\title{
Microfluidics and Biosensors
}

\section{Empirical Model for Identifying Protein Concentrations in Wound using Cyclic Voltammetry}

\author{
Yomna EISaboni ${ }^{1}$, John A. Hunt ${ }^{23}$, Christine Moffatt ${ }^{4}$ and Yang Wei ${ }^{1}$ \\ ${ }^{1}$ Smart wearable research group, Department of Engineering, School of Science and Technology, Nottingham Trent University, NG11 \\ 8NS Nottingham, UK \\ ${ }^{2}$ Medical Technologies Innovation Facility, Nottingham Trent University, Nottingham, NG11 8NS, U.K \\ ${ }^{3}$ College of Biomedical Engineering, China Medical University, Taichung 40402, Taiwan \\ ${ }^{4}$ Skin Integrity, Institute care excellence, Nottingham University Hospital, Nottingham, NG5 1PB, UK
}

\begin{abstract}
The establishment of an accurate and efficient method for the sensitive detection of protein concentrations in fluids using miniaturized electrochemical sensors is presented. As protein levels in wounds can be utilized to assess the status and severity of a wound and determine the best course of treatment, real time quantification is potentially extremely valuable. The experimental methodology for monitoring protein concentrations using screen printed carbon electrodes (SPCEs) that requires minimal sample size (as small as $80 \mu \mathrm{L}$ ) for each measurement is presented. The technique was modelled and implemented using bovine serum albumin (BSA) concentrations from 0.3 to $30 \mathrm{mg} / \mathrm{ml}$ and was used to detect changes in concentration. The results demonstrated a good stability and reproducibility when making measurements on different protein concentrations. This technique was tested and verified using two different types of screen printed carbon electrodes (SPCEs), while both consist of three electrode electro-chemical cell, one has an added Poly-L-Lysine coating to anchor protein and improve efficiency. The objective of this work was to establish for the first time a device with the necessary mathematical model that can be used by clinicians to assess the severity of the wound through measuring the protein concentration.
\end{abstract}

\section{INTRODUCTION}

Light weight low power disposable electrochemical biosensor technologies are becoming increasingly utilized in healthcare as they facilitate patient independent living and help support remote monitoring of vital signals, falls and movement. In the case of skin wounds they are utilized for chronic or slow healing wounds with diabetic patients [1]. Improving remote monitoring techniques not only improves treatment but increases the quality of life for the patient and also reduces the need for face to face consultations and treatments with healthcare providers. The cost of treating wounds is constantly a critical issue as it is estimated to account for at least $3 \%$ of the total healthcare expenditure in most developed countries [2] and in 2012 the UK spent approximately $£ 5.1$ billion on costs associated with clinical wound care management [3].

One approach to assess the severity of wounds [4] is through testing the $\mathrm{pH}$ level. It has been measured extensively in the past and is considered a good indicator of wound state, because the $\mathrm{pH}$ of the surface of human skin is usually slightly acidic and lies in the range of 4.2 - 5.6. Once $\mathrm{pH}$ starts to rise, bacteria survival and proliferation increases and infection can become established resulting in delayed healing or complete lack of wound healing [5]. However, due to the composition of the environment of skin with open wounds $\mathrm{pH}$ sensors can suffer from interference effects on the sensing performance from ions present in wound fluids [6].

Another qualitative biomarker that can be indicative of wound severity conditions is protein concentrations, for which a concentration $>15 \mathrm{mg} / \mathrm{ml}$ is indicative of an inflamed wound [7]. The normal protein level in a healing wound is $9 \mathrm{mg} / \mathrm{ml}$, contrasted against levels of 35 $\mathrm{mg} / \mathrm{ml}$ for chronic slow healing wounds [8]. One successful approach in detecting protein concentrations is using electrochemical sensors

Corresponding author: Y. EISaboni (e-mail: yomna.elsaboni@ntu.ac.uk).
[9]. Different types of empirical testing that have been conducted in the past include impedance spectroscopy, potentiometry and amperometry [10] to detect glucose and nucleic acids, as well as protein [11-13]. In one recent study impedance spectroscopy was used to study the protein concentration in pasteurized milk focusing only on utilizing a potentiostat [14]. Electro-chemical sensing methods can provide high sensitivity, they are relatively easy to implement and setup and most importantly are suitable for miniaturization and for incorporation into wearable garments and accessories [9].

One method of fabricating miniaturized flexible biosensors is by screen printing the electrodes. Screen printing electrodes is proving to be useful not only because they provide reliable miniaturized electronics for data capture and signal detection, but they are also suitable for the mass production of reproducible electrodes at commercially realistic production costs [15]. The technology used in fabricating this type of electrodes can also improve patient independence by integrating a wireless module and readout electronics to monitor and record data remotely [4]. Importantly, their structure and geometry is extremely flexible, making this technique more adaptable than conventional electro-chemical cells that use at least two metal rods (commonly silver) as electrodes immersed in the solution under test in a glass container.

To develop an empirical testing methodology, one common material that is easy to make and produce precise samples often used as a protein concentration standard is bovine serum albumin (BSA) [16]. The use of BSA as a protein source specifically when testing electrochemical sensors has been previously utilized by others $[12,17]$. BSA is useful because human albumin is the most abundant protein in blood plasma and represents $50 \%$ of total protein present in wounds, which makes it a good representative of protein in blood [18]. Not to mention that its concentration is easily standardized and altered precisely to evaluate the detection range. BSA dissolved in water was the binary protein solution used to present protein in a water based liquid. For 
the first time in the literature, this research proposes a mathematical model based on empirical measurements conducted on different concentrations of BSA solution to mimic the protein concentration present in a slowly healing wound. The sensors currently used to conduct measurements consist of three electrodes: counter, working and reference, printed using carbon ink. This model can be used to predict protein concentration by conducting a series of empirical cyclic voltammetery experiments and recording the output current produced at the working electrode.

This article was structured as follows. Section II described the experiment design and methodology. Section III presented experimentally derived results and also how a model was established to estimate the protein concentration in a fluid sample. Section IV was the conclusion.

\section{EXPERIMENTAL SETUP}

\section{A. Materials and Instrumentation preparation}

1) Materials: Bovine serum albumin (BSA) powder as it is composed of 583 amino acids and is considered as a standard for protein quantification which was prepared using heat shock fractionation and purchased from Sigma-Aldrich (Steinheim, Germany). 8 samples of BSA were prepared with different concentrations and diluted using deionized water: $30,23,18,11,7,3,1,0.3 \mathrm{mg} / \mathrm{ml}$. The range of concentrations were chosen based on the concentration limit of the BSA powder under test which is $40 \mathrm{mg} / \mathrm{ml}$. Supermarket fresh pasteurized semi skimmed milk fluid available at $34 \mathrm{mg} / \mathrm{ml}$ was used to cross validate the proposed empirical model at three concentrations 34,24 , and $4 \mathrm{mg} / \mathrm{ml}$, diluted as required using deionized water.

2) Instruments: Screen-printed carbon electrodes (SPCEs) (Metrohm Dropsens, Oviedo, Spain) had a $4 \mathrm{~mm}$ diameter carbon working electrode, a carbon counter electrode, and a silver/silver chloride $(\mathrm{Ag} / \mathrm{AgCl})$ reference electrode. The structure of the SPCEs is shown in Fig. 1[19]. These sensors are suitable for both; inserting a micro-volume of fluid or dipping them in solutions. The electrodes sit on a ceramic substrate with the dimensions: : L33 x W10 x H0.5 $\mathrm{mm}$. The two types of SPECEs used for comparison are : type 1 is named 110 and type 2110 PLYS (Poly-L-Lysine coated).

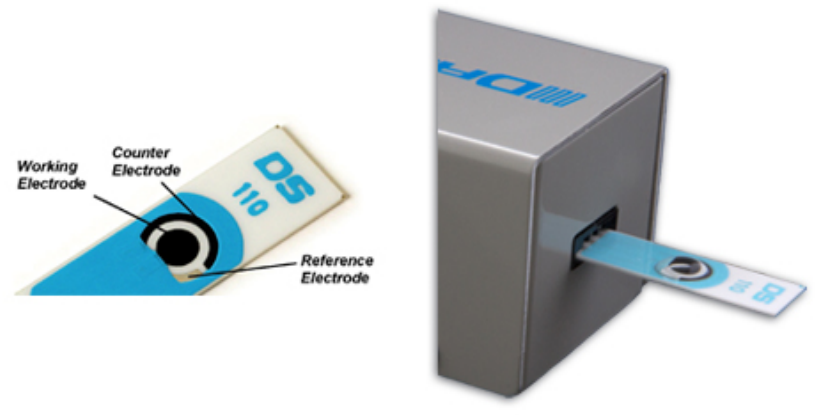

Fig. 1. Structure of SPCE and setup used [19]

\section{B. Experimental Procedure}

Conducting cyclic voltammetry was used to analyze the redox reaction on different protein solutions and record the oxidation peak
TABLE 1. Cyclic voltammetry measurement parameters

\begin{tabular}{|c|c|}
\hline Parameter & Value \\
\hline Measurement type & CV staircase \\
\hline Scan rate & $0.08 \mathrm{~V} / \mathrm{s}$ \\
\hline Start potential & $0.0 \mathrm{~V}$ \\
\hline Step & $0.0025 \mathrm{~V}$ \\
\hline Upper vertex potential & $1.6 \mathrm{~V}$ \\
\hline Lower vertex potential & $-1.2 /-1.6 \mathrm{~V}$ \\
\hline
\end{tabular}

obtained using an AUTOLAB potentiostat device. The set parameters of Metrohm AUTOLAB software are listed in Table 1. The sensors consisted of three electrodes: counter, working and reference screen printed using carbon and silver inks known as DropSens. They were screen printed and had a 2D strip structure and an overall dimension of $3.4 \times 1.0 \times 0.05 \mathrm{~cm}$. Two types were used and tested to compare and validate the experimental methodology proposed. The former design was termed 110 and the latter referred to as 110 PLYS by the manufacturer. Both SPCEs had the same features, but the latter had a coating on top that provided a protein anchoring mechanism. These electrodes only required a very small sample volume (which could be as small as $4 \mu \mathrm{L}$ ), the carbon layer acted as a barrier and prevented the oxidation of the silver electrodes.

The setup used to conduct measurements is provided in Fig. 1, where the sensor is enclosed in a high humidity environment to prevent drops from evaporating. To achieve this, the sensors were surrounded by water containers which also contained ice to minimize any change in temperature with that further changes in the rate of evaporation. Control of the environment was key in order to continuously monitor the sample over a period of four hours.

The empirical experimentation was divided into two main stages:

1) Stage 1: $: 30 \mathrm{mg} / \mathrm{ml}$ of BSA was tested using the two types of SPCEs. $30 \mathrm{mg} / \mathrm{ml}$ is the highest concentration under test and is the most indicative of protein in fluid. In each test, a cyclic voltammetry measurement was taken continuously over a period of 270 minutes as illustrated in Fig. 2. It was noticed that after 270 minutes, minimal changes in response occurred, therefore it was not considered as the aim of this experiment was to observe any changes to the redox reaction.

2) Stage 2: :Different concentrations of each test solution were tested once using both types of SPCEs. Oxidation current peaks were obtained for each concentration $(30,25,20,18,15,11,7$, $3,1,0.3 \mathrm{mg} / \mathrm{ml}$ ) and used to determine the relationship between concentration and output current observed at oxidation. Additionally, to cross validate the model, milk was tested, analyzed and compared in the same way.

\section{RESULTS AND DISCUSSION}

\section{A. SPCE type comparison}

Measurements were recorded continuously over a period of 270 minutes, Fig. 2 using both SPCE type 1 and type 2. The oxidation peak stabilized at approximately 30 minutes. The graph 2 (A) are the results obtained using the SPCE type 1 (original dropsens design), while 2(B) 
are for type 2 (which has an added Poly-L-Lysine coating). Because Type 2 captured proteins more effectively, the current recorded at oxidation peak changes considerably less between the different runs in comparison with Type 1 by almost half the current $(0.04 \mathrm{~mA}$ and $0.08 \mathrm{~mA}$ ). It was important to note that the potential applied was different between the two types of SPCEs, because type 2 required a higher negative potential for the reduction reaction to complete. However, because the current response observed when using Type 2 is more stable, in the future, type 2 is better equipped to conduct this experiment despite the voltage potential needed to complete the measurement.

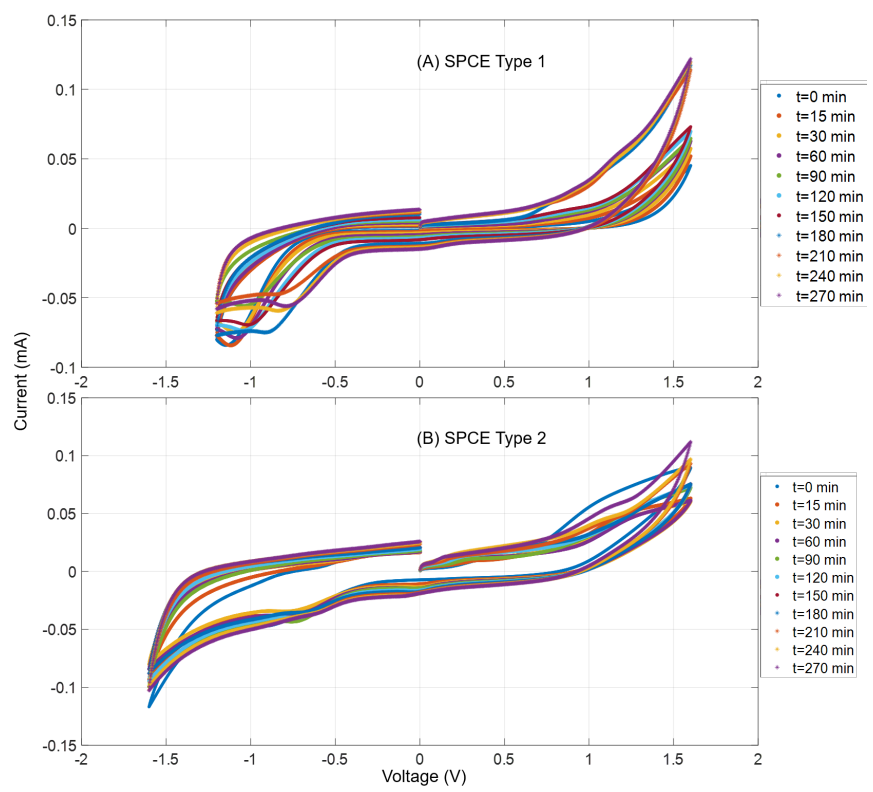

Fig. 2. Continuous measurement of BSA using SPCE (A) Type 1 and (B) Type 2

Fig. 3 shows a bland-altman plot comparing the oxidation peaks of $30 \mathrm{mg} / \mathrm{ml}$ BSA solution using both SPCEs to demonstrate their similarity. This also validated the method being implemented to measure protein concentrations, because for the same sample the two SPCEs presented a level of similarity as the points on the graph always existed within the double standard deviation boundaries.

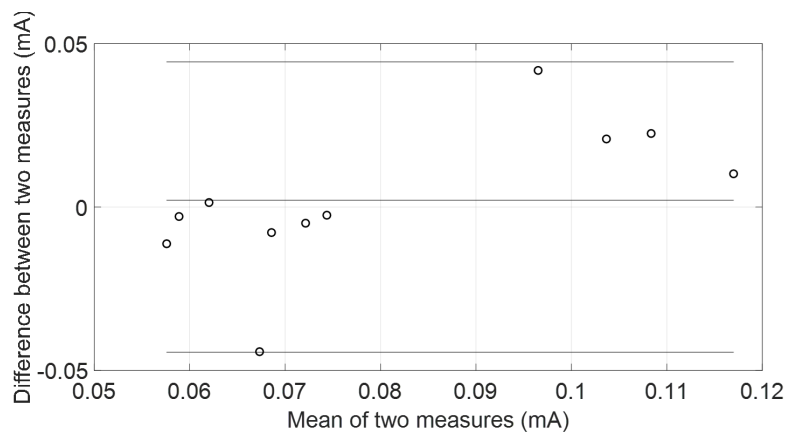

Fig. 3. Comparison between the two types of SPCE

\section{B. Empirical model}

Equation (1) was established based on the relationship observed between protein concentrations in fluid and current produced during the redox reaction. In the equation, current peak $(\mathrm{mA})$, protein concentration $(\mathrm{mg} / \mathrm{ml})$, rate of increase in output current based on change in concentration for each solution and expected current peak with zero concentration $(\mathrm{mA})$ are denoted by $I_{c o n}, M_{\text {pro }}, R, I_{0}$.

$$
M_{\text {pro }}=\left(I_{\text {con }}-I_{0}\right) / R
$$

To illustrate how this formula could be implemented and demonstrate its ability to identify and distinguish concentrations, an example was presented in Table 2 where varying BSA concentrations were prepared and were chosen to validate the sensitivity that can be captured by the SPCEs and the corresponding maximum peak obtained during oxidation measured and recorded. The values calculated are based on the average of three measurements repeated for each solution.

TABLE 2. Parameters obtained and calculated for BSA

\begin{tabular}{|c|c|c|c|c|}
\hline SPCE & Conc. $(\mathrm{mg} / \mathrm{ml})$ & $I_{c o n}(\mathrm{~mA})$ & Cal. $R$ & Cal. $I_{0}(\mathrm{~mA})$ \\
\hline & 30 & 0.071 & & \\
Type 1 & 3 & 0.02 & 0.025 & 0.16 \\
& 0.3 & 0.01 & & \\
\hline & 30 & 0.093 & & \\
Type 2 & 3 & 0.029 & 0.026 & 0.16 \\
& 0.3 & 0.011 & & \\
\hline
\end{tabular}

In addition to the concentrations listed in Table 2,8 additional concentrations from $0.3-30 \mathrm{mg} / \mathrm{ml}$ were also tested and recorded. The values are then plotted on a graph and the best fit line is estimated. The calculated rate is based on the slope of the best fit line of all the measured values Fig. 4. Moreover, $I_{0}$ was also deduced from the best fit line for each SPCE. The computed $R$ and $I_{0}$ for both SPCEs are in Table 2.

To cross validate the model proposed, fresh milk was used as a different source of protein fluid to test at different concentrations. The same methodology was repeated but with different concentrations to the ones used in Table 2. To calculate $P_{0}$ and $I_{0}$, the experiments were only conducted using SPCE type 2, because as been presented in Fig. 2, type 2 stabilized quicker and therefore are more efficient to be used for this model.

\section{TABLE 3. Parameters obtained and calculated for Milk}

\begin{tabular}{|c|c|c|c|c|}
\hline SPCE & Conc. $(\mathrm{mg} / \mathrm{ml})$ & $I_{\text {con }}(\mathrm{mA})$ & Cal. $R$ & Cal. $I_{0}(\mathrm{~mA})$ \\
\hline & 34 & 0.6 & & \\
Type 2 & 17 & 0.4 & 0.014 & 0.16 \\
& 4 & 0.2 & & \\
\hline
\end{tabular}

The computed value of $R$ indicated that the rate at which the current changed with varying protein concentration was slightly slower, and therefore the sensor was less sensitive to any change, despite the fact that the oxidation peak was much higher than the range of peaks obtained for BSA. Moreover, the $I_{0}$ of milk was almost the same as BSA, which verified the model. This was because $I_{0}$ is a computed estimate of the peak obtained with zero concentration and should remain in the same range $(0.16)$. 


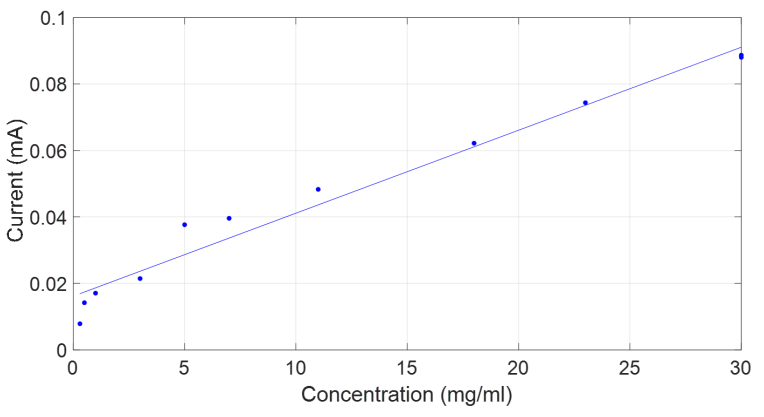

Fig. 4. Predicted relationship between protein concentration and output current

This research can be used by first identifying the parameters $R$ and $I_{0}$ and then by using the formula derived from the best fit line shown in Fig. 4, an unknown protein concentration can be determined by plotting the IV of a cyclic voltammetry experiment conducted on liquids and capturing output current obtained at the oxidation peak. In the case of BSA where $R$ and $I_{0}$ estimated to be 0.026 and 0.16 respectively, the parameters can be substituted in equation 2 . In the case of milk, $R$ is 0.014 instead of 0.026 . Therefore, in the future an unknown concentration of both BSA and milk can be identified using the equations (2) by observing the oxidation peak current obtained through cyclic voltammetry conducted on SPCE Type 2.

$$
M_{\text {pro }}=\left(I_{\text {con }}-0.16\right) /(0.026)
$$

\section{CONCLUSION}

A new approach to analyzing and modelling the relationship between the amount of protein dissolved in a water based liquid with the output current observed at the working electrode of an electrochemical cell when conducting cyclic voltammetry has been demonstrated and experimentally validated. A methodology for comparing and identifying protein concentrations has been outlined and an empirical model established for two types of protein solutions, BSA and fresh milk. BSA was used to establish the methodology of determining the protein concentration from the measured output current value, while the latter solution was used to cross validate the established model. Furthermore, two types of SPCEs were used when conducting measurements to validate the proposed empirical methodology. This model can be used to predict and assess the condition of a wound through conducting minimally invasive measurements in real time directly on a wound.

\section{ACKNOWLEDGMENT}

This research was funded by Nottingham Trent University strategic research theme, Medical Technologies and Advanced Materials, and supported by Kymira Ltd.

\section{REFERENCES}

[1] J. Phair, L. Newton, C. McCormac, M. F. Cardosi, R. Leslie, and J. Davis, "A disposable sensor for point of care wound ph monitoring," Analyst, vol. 136, pp. 4692-4695, 2011. [Online]. Available: http://dx.doi.org/10.1039/C1AN15675F

[2] M. Olsson, K. Jarbrink, U. Divakar, R. Bajpai, Z. Upton, A. Schmidtchen, and J. Car, "The humanistic and economic burden of chronic wounds: A systematic review," Wound Repair and Regeneration, vol. 27, pp. 114-125, 012019.
[3] J. F. Guest, N. Ayoub, T. McIlwraith, I. Uchegbu, A. Gerrish, D. Weidlich, K. Vowden, and P. Vowden, "Health economic burden that different wound types impose on the uk's national health service," International Wound Journal, vol. 14, no. 2, pp. 322-330, 2017. [Online]. Available: https://onlinelibrary.wiley.com/doi/abs/10.1111/iwj.12603

[4] N. Tang, Y. Zheng, X. Jiang, C. Zhou, H. Jin, K. Jin, W. Wu, and H. Haick, "Wearable sensors and systems for wound healing-related ph and temperature detection," Micromachines, vol. 12, no. 4, p. 430, 2021. [Online]. Available: https://www.mdpi.com/2072-666X/12/4/430

[5] S. Ono, R. Imai, Y. Ida, D. Shibata, T. Komiya, and H. Matsumura, "Increased wound ph as an indicator of local wound infection in second degree burns," Burns, vol. 41, no. 4, pp. 820-824, 2015. [Online]. Available: https://www.sciencedirect.com/science/article/pii/ S0305417914003593

[6] L. Manjakkal, D. Szwagierczak, and R. Dahiya, "Metal oxides based electrochemical ph sensors: Current progress and future perspectives," Progress in Materials Science, vol. 109, p. 100635, 2020. [Online]. Available: https://www.sciencedirect.com/science/article/pii/ S0079642519301173

[7] L. E. Edsberg, J. T. Wyffels, R. Ogrin, B. C. Craven, and P. Houghton, "A pilot study evaluating protein abundance in pressure ulcer fluid from people with and without spinal cord injury," The Journal of Spinal Cord Medicine, vol. 38, no. 4, pp. 456-467, 2015.

[8] C. Legendre, C. Debure, S. Meaume, C. Lok, J. L. Golmard, and P. Senet, "Impact of protein deficiency on venous ulcer healing," Journal of Vascular Surgery, vol. 48, no. 3, pp. 688-693, 2008. [Online]. Available: https://www.sciencedirect.com/science/article/pii/S0741521408005909

[9] P. Salvo, V. Dini, A. Kirchhain, A. Janowska, T. Oranges, A. Chiricozzi, T. Lomonaco, F. Di Francesco, and M. Romanelli, "Sensors and biosensors for c-reactive protein, temperature and ph, and their applications for monitoring wound healing: A review," Sensors, vol. 17, no. 12, p. 2952, 2017. [Online]. Available: https://www.mdpi.com/1424-8220/17/12/2952

[10] M. Mathew, S. Radhakrishnan, A. Vaidyanathan, B. Chakraborty, and C. Rout, "Flexible and wearable electrochemical biosensors based on two-dimensional materials: Recent developments," Analytical and Bioanalytical Chemistry, pp. 1 - 36, 2020.

[11] P. Seumo, C. Nanseu-Njiki, E. Ngameni, R. Danielsson, T. Arnebrant, and T. Ruzgas, "Determination of total protein concentration in solution using gold electrode modified with silver nanoparticles," Electroanalysis, vol. 27, 122014.

[12] D. Zhang, Y. Lu, Q. Zhang, L. Liu, S. Li, Y. Yao, J. Jiang, G. L. Liu, and Q. Liu, "Protein detecting with smartphone-controlled electrochemical impedance spectroscopy for point-of-care applications," Sensors and Actuators B: Chemical, vol. 222, pp. 994-1002, 2016. [Online]. Available: https: //www.sciencedirect.com/science/article/pii/S0925400515303488

[13] M. Pohanka and P. Skládal, "Electrochemical biosensors-principles and applications," Journal of Applied Biomedicine, vol. 6, 072008.

[14] A. Lopes, J. Tenreiro Machado, E. Ramalho, and V. Silva, "Milk characterization using electrical impedance spectroscopy and fractional models," Food Analytical Methods, vol. 11, 032018.

[15] P. Havranova, L. Fojt, L. Kejik, T. Sikola, M. Fojta, and A. Danhel, "Electrodeposition of silver amalgam particles on screen-printed silver electrodes in voltammetric detection of 4-nitrophenol, bovine serum albumin and artificial nucleosides dtpt3 and d5sics," Sensors and Actuators B: Chemical, vol. 340, p. 129921, 2021. [Online]. Available: https://www.sciencedirect.com/science/article/pii/S0925400521004901

[16] H. J. Lee and J.-X. Cheng, "5 - label-free stimulated raman scattering imaging of neuronal membrane potential," in Neurophotonics and Biomedical Spectroscopy, ser. Nanophotonics, R. R. Alfano and L. Shi, Eds. Elsevier, 2019, pp. 107-122. [Online]. Available: https: //www.sciencedirect.com/science/article/pii/B9780323480673000056

[17] D. Compagnone, Q. Al-Khafaji, M. Harris, S. Tombelli, S. Laschi, A. Turner, M. Mascini, and G. Marrazza, "An electrochemical immunoassay for her2 detection," Electroanalysis, vol. 24, 042012.

[18] P. Caraceni, M. Tufoni, and M. Bonavita, "Clinical use of albumin," Blood transfusion = Trasfusione del sangue, vol. 11 Suppl 4, pp. s18-25, 092013.

[19] Accessed on 05.10.2021. [Online]. Available: https://www.dropsens. com/en/faqs_dropsens.html 